\title{
Status and perspective of FARCOS: A new correlator array for nuclear reaction studies
}

\author{
E.V. Pagano ${ }^{1,2}$, L. Acosta ${ }^{3,4}$, L. Auditore ${ }^{5}$, C. Boiano ${ }^{6}$, \\ G. Cardella $^{3}$, A. Castoldi ${ }^{7}$, M. D'Andrea $^{3}$, D. Dell'aquila ${ }^{8}$, \\ E. De Filippo ${ }^{3}$, S. De Luca $^{5}$, F. Fichera ${ }^{3}$, L. Francalanza ${ }^{8}$, \\ N. Giudice ${ }^{1,3}$, B. GNOFFo ${ }^{3}$, A. Grimaldi ${ }^{3}$, C. Guazzoni ${ }^{6,7}$, \\ G. Lanzalone ${ }^{2,9}$, I. Lombardo ${ }^{8}$, T. Minniti ${ }^{11,12}{ }^{3}$, S. Norella ${ }^{5}$,

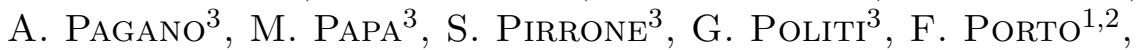 \\ L. Quattrocchi ${ }^{5}$, F. Rizzo ${ }^{1,2}$, P. Russotto ${ }^{3}$, G. Saccá ${ }^{3}$,
}

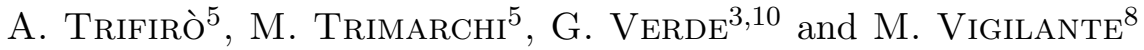

${ }^{1}$ Università di Catania, Catania, Italy ${ }^{2}$ INFN - Laboratori Nazionli del Sud, Catania, Italy

${ }^{3}$ INFN, Sezione di Catania, Catania, Italy

${ }^{4}$ Istituto de Física, Universidad Nacional Autónoma de México, México City, México

${ }^{5}$ Dipartimento di Fisica e Scienze della Terra, Università di Messina, INFN, gr. collegato di Messina, Messina, Italy

${ }^{6}$ INFN, Sezione di Milano, Milano, Italy

${ }^{7}$ Politecnico di Milano, Dipartimento di Elettronica, Informazione e Bioingegneria, Milano, Italy

${ }^{8}$ INFN, Sezione di Napoli, Dipartimento di Fisica, Università di Napoli Federico II, Napoli, Italy

${ }^{9}$ Università di Enna Kore, Enna, Italy

${ }^{10}$ Institute de Physique Nucléaire Orsay, Orsay, France

${ }^{11} \mathrm{CNR}$ - Istituto per I Processi Chimico-Fisici, Messina, Italy

${ }^{12}$ ISIS Facility, Science and Technology Facilities Council, Rutherford Appleton Laboratory, Oxfordshire, UK 


\begin{abstract}
The experimental investigation of Heavy Ion reactions at Fermi energies requires an accurate measurement of observables, such as linear momentum and energy of the detected particles. In order to address this problem, dedicated and flexible correlator arrays are useful tools to be coupled with $4 \pi$ detectors. One of these arrays is FARCOS, presently under construction at the INFN Sezione di Catania and Laboratori Nazionali del Sud (LNS).
\end{abstract}

The study of dynamics of nuclear reactions in heavy ion (HI) collisions at Fermi energy $(20 \mathrm{MeV} / \mathrm{u}<\mathrm{E} / \mathrm{A}<200 \mathrm{MeV} / \mathrm{u})$ is an active area in nuclear science [1]. In this energy region, an impressive range of physical phenomena occurs within large time-scales (from 10 to $1000 \mathrm{fm} / \mathrm{c}$ ) which are revealed through imprints on space-time and particle-particle correlations [2]. In particular, nuclear matter can experiences variation with respect to the saturation density $\rho_{0}\left(\approx 0.17\right.$ nucleon $\left./ \mathrm{fm}^{3}\right)$ [3]. Exotic nuclei can be also produced and studied in close connection with nuclear astrophysics [4]. With the availability of new-generation radioactive ion-beam facilities (RIB), like for instance SPES at LNL, SPIRAL II at GANIL, FAIR at GSI or FRIB at MSU or the possible upgrade of the heavy ion CS facility in Catania, such studies assume renewed interest. Experimentally, large efforts has been devoted in the last two decades in order to achieve high energy and angular resolution of dedicated detection systems [5]. In this paper we briefly describe the next-generation correlator FARCOS (Femtoscope ARray for COrrelation and Spectroscopy) for reaction and spectroscopic studies [6]. FARCOS is a modular array of telescopes arranged in a single cluster, each one consisting of three detection stages. The first two stages are two Double Sided Silicon Strip Detectors (DSSSD) of active area $6.4 \times 6.4 \mathrm{~cm}^{2}$ with 32 vertical strips in the front side and 32 horizontal strips in the back side and are $300 \mu \mathrm{m}$ and $1500 \mu \mathrm{m}$ thick respectively. The last stage consists of four CsI(Tl) crystals $\left(3.2 \times 3.2 \mathrm{~cm}^{2}\right)$ of $6 \mathrm{~cm}$ of thickness read by a photodiode $\left(18 \times 18 \mathrm{~mm}^{2}\right)$. We show in Fig. 1 a typical close assembling of one cluster.

Particle identification is performed by $\Delta \mathrm{E} / \mathrm{E}$, pulse shape(PSD) and Time of Flight (TOF) techniques. Portability and modularity are important characteristics making feasible the easy coupling with $4 \pi$ detectors, other correlators and/or magnetic spectrometers. Digital Signal Acquisition (DSA) is also envisaged. We used, preliminary, in the electronic front-end, a preamplifier (PAC) derived from an improved versions of CHIMERA-type PACs developed to minimize the electronics noise and to optimize TOF performances [7]. So, the read-out of silicon strips was performed by using a compact housing $\left(86 \times 80 \times 10 \mathrm{~mm}^{3}\right.$ (NPA-16FL), 98x80x15 mm (NPA- $^{3}$ 


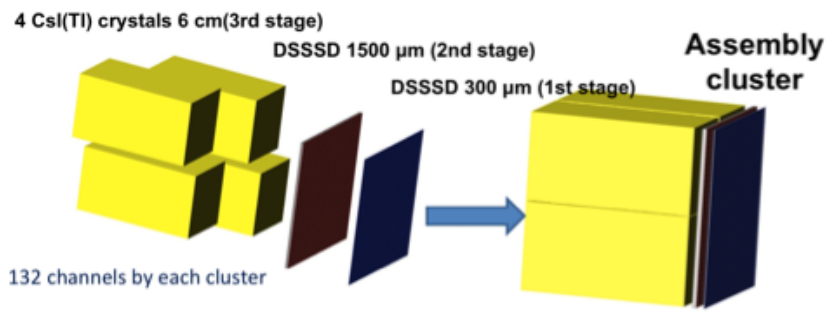

Figure 1: schematic view of a cluster of FARCOS

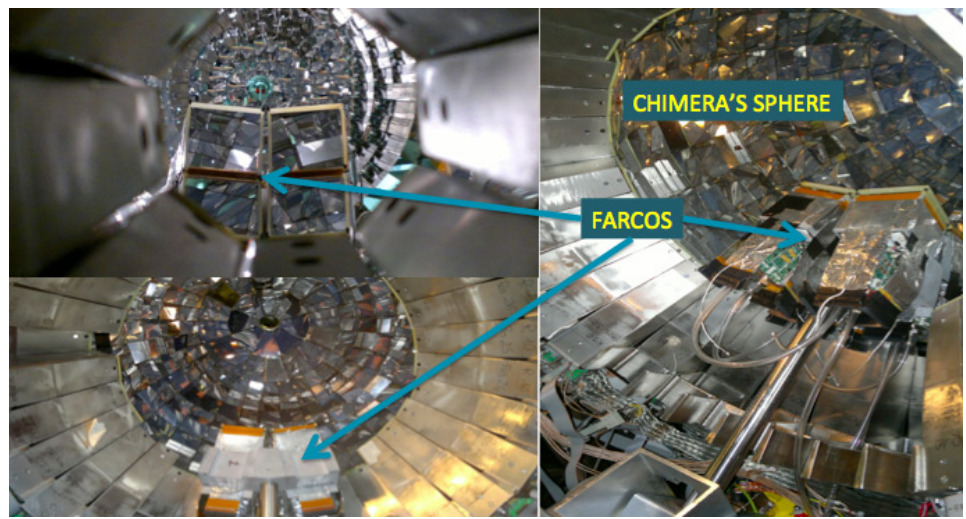

Figure 2: The INKIISSY experimental setup. ${ }^{124} \mathrm{Xe}+{ }^{64} \mathrm{Zn} @ 35 \mathrm{MeV} / \mathrm{A}$ was the studied reaction.

$16 \mathrm{FE})$ ) allocating 32-channels PACs with low power consumption $(<900$ $\mathrm{mW}$ for 32 channels). FARCOS prototype was tested and coupled for the first time with the $4 \pi$ CHIMERA [8] in the INKIISSY (INverse KInematic ISobaric System) experiment [9] in Catania on April 2013 with beams delivered by INFN-LNS facility. FARCOS covered the range of polar and azimuthal opening, $\theta_{l a b} \approx 16^{\circ} \div 44^{\circ}$ and $\Delta \phi \approx 75^{\circ}$, respectively, corresponding to a solid angle coverage of $\approx 262 \mathrm{msr}$ (see Fig. 2). As a consequence, a portion of CHIMERA was shielded by FARCOS telescopes, thus giving the opportunity to investigate about the detection of neutrons [10].

In reference $[6]$, the $\Delta \mathrm{E} / \mathrm{E}$ identification procedures in the first two DSSSD stages (see Fig. 3) have been already discussed and a good isotopic identification was shown for IMFs up to atomic number $Z=10$. Good results have been also obtained by applying PSD method in $\mathrm{CsI}(\mathrm{Tl})$ for light charged particles up to lithium and ${ }^{8} \mathrm{Be}$ (see Fig. 4). 


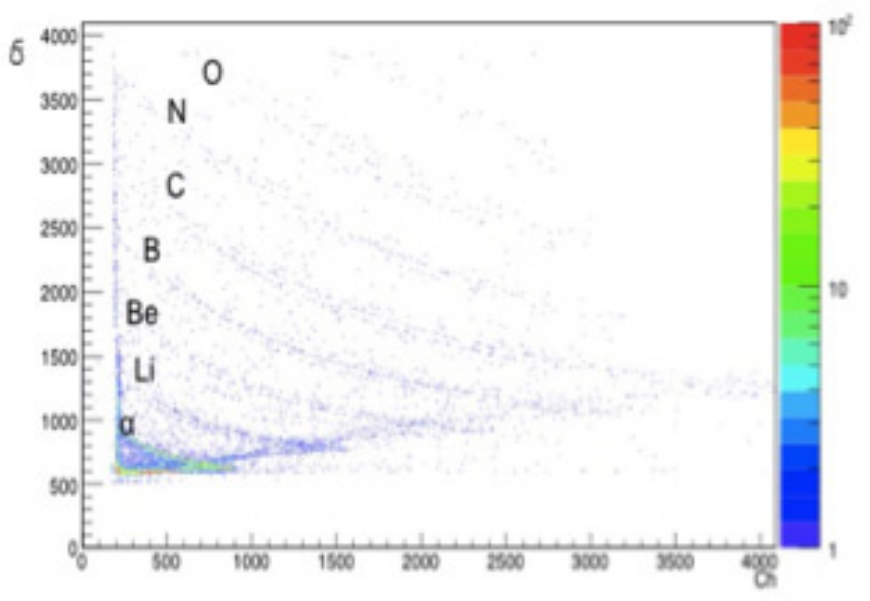

Figure 3: $\Delta \mathrm{E} / \mathrm{E}$ identification matrix of two strips between the first and the second DSSSD stages (INKIISSY experiment).
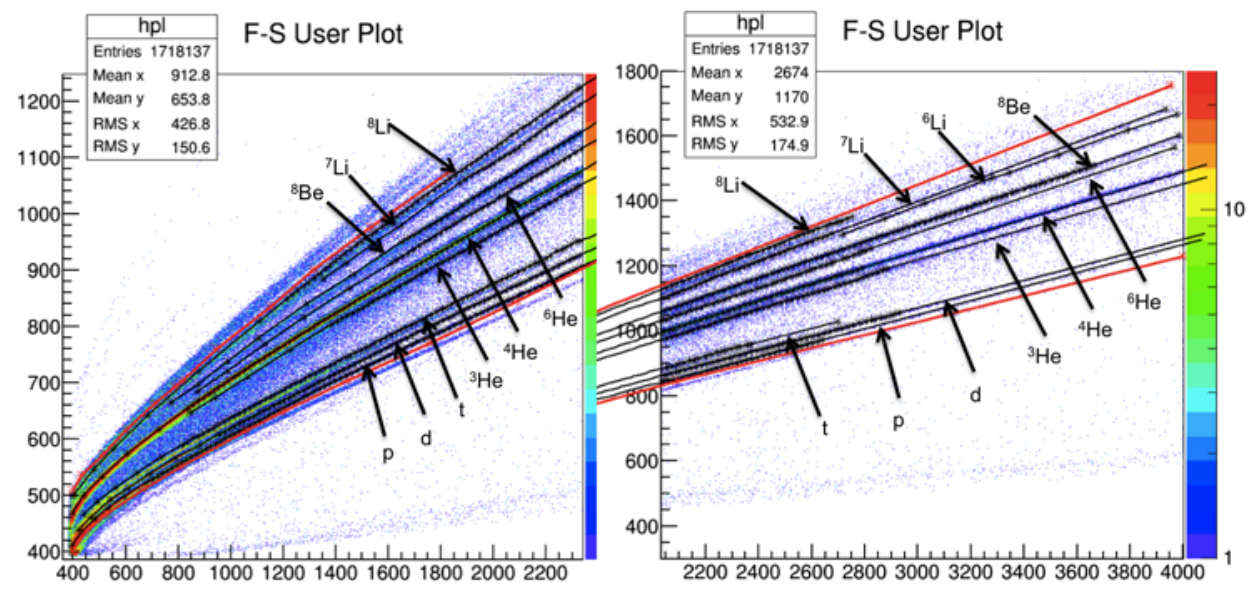

Figure 4: Typically PSD analysis identification in a CsI(Tl) of FARCOS (INKIISSY experiment). 

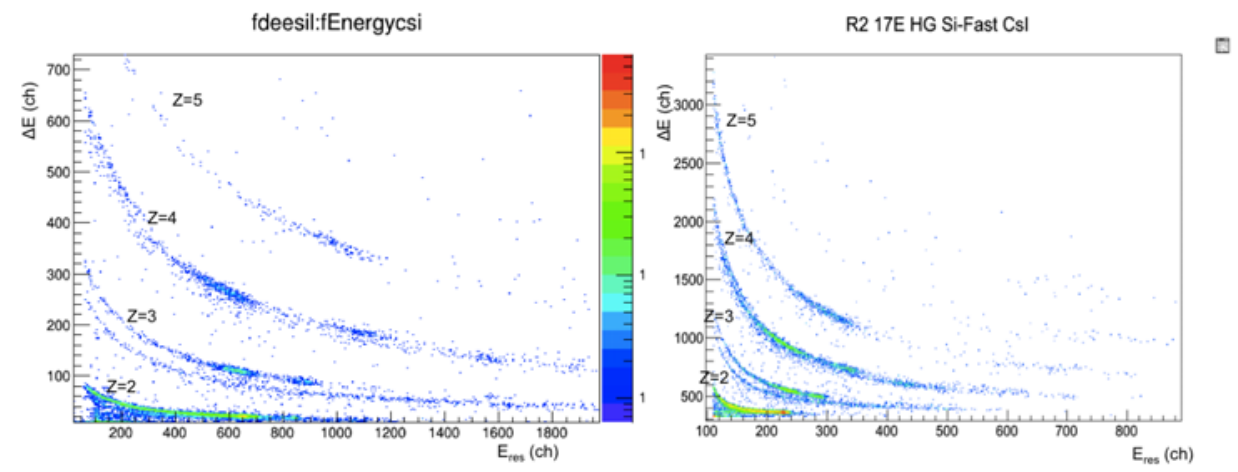

Figure 5: comparison between two $\Delta \mathrm{E} / \mathrm{E}$ identification matrix obtained in the reaction ${ }^{16} \mathrm{C}+{ }^{12} \mathrm{C} @ 40 \mathrm{MeV} / \mathrm{A}$ (CLIR experiment) from two telescopes of CHIMERA detector of the ring two. Left: signals processed by GET. Right: signals processed by standard CHIMERA electronic

The GET (Generic Electronic for TPC) electronics for read-out and DAQ, was also tested. GET is an compact system designed to handle a very large number of independent electronic channels (more than 30kch). The innovative performances such as high versatility and compactness make the GET project a good candidate for FARCOS array (132 channels for each cluster allocated in a small volume, about $\left.7 \times 7 \times 10 \mathrm{~cm}^{3}\right)$. GET is based on one chip, AGET, processing 64 independent channels. Each channel consists of a preamplifier, a filter, a discriminator and an array of 512 capacitors having sampling frequency ranging from 1 to $100 \mathrm{MHz}$. Four AGET chips are allocated on a ASAD board for the digitalization of 256 channels. A block of four ASADs (1024 chs) can be read-out by a single CoBo. Up to $10 \mathrm{CoBo}$ boards can be house in the $\mu \mathrm{TCA}$ crate. In this same crate, a MUTANT card is used for triggering, synchronizing different CoBos and for managing inter-communications among different devices [11]. Silicon detectors and $\mathrm{CsI}(\mathrm{Tl})$ crystals were tested by comparing the CHIMERA and the GET DAQ systems (see Fig. 5). The result of this comparison is quite satisfactory.

Milestones of FARCOS construction are briefly summarized in the following. The test of GET electronics will be completed within the second semester of year 2015, while in the first semester of 2016 it is expected to test the new ASIC preamplifiers designed by INFN and Politecnico of Milan. In 2016 six new clusters will be assembled; in 2017 and in 2018 four new clusters for each year are planned to be added to the array. The final 
configuration of 20 clusters of FARCOS array is planned to be in operation on 2019.

In conclusion, we have tested performances of four clusters of the FARCOS prototype. The obtained results are quite satisfactory and fit the requirements for new generation correlators for charged light particles and fragments, in HI physics at intermediate energies. On 2015 year the FARCOS prototype will be used in two experiments at LNS, i.e., CLIR and SIKO. The use of the detector is also envisaged in various Letters of Intent submitted to the new radioactive Ion beam facility, SPES, that is under construction at INFN-Laboratori Nazionali di Legnaro.

\section{References}

[1] Proceedings of the IWM-EC (2014), Catania, (Italy), Vol.108, pub. by the Italian Physical Society, Bologna, Eds. B. Borderie, R. Bougault, A. Pagano, S. Pirrone and G. Politi. www.epjconferences.org/articles/epjconf/abs/2015/07/contents/contents.html.

[2] S. Koonin, Phys. Lett. B 7043 (1977)

[3] Bao-An Li et al., Phys. Rev. C 71044604 (2005)

[4] V. Baran, M. Colonna, V. Greco, M. Di Toro, Physics Reports, Volume 410, Issues 56, May 2005, Pages 335-466

[5] R.T. De Souza, N. Le Neindre, A. Pagano and K. H. Schmidt, Eur. Phys. J. A 3, 275-291 (2006).

[6] E. V. Pagano et al., EPJ Web of Conferences 88, 00013 (2015), and contribution in Proceedings ISTROS 2015 International Conference (in press).

[7] C. Boiano, et al., IEEE Trans. On Nucl. Science 511931 (2004)

[8] E. De Filippo, A.Pagano, European Physical Journal A50 (2014) 32, and reference therein.

[9] P. Russotto et al., Phys. Rev. C 91, 014610 (2015).

[10] L. Auditore, et al., EPJ Web of Conferences 88, 01001 (2015).

[11] E.Pollacco et al., Phys. Procedia 37 (2012) 1799-1804. 